Bundesgesundheitsbl 2013 · 56:38-41 DOI 10.1007/s00103-012-1591-3

Online publiziert: 19. Dezember 2012

c) Springer-Verlag Berlin Heidelberg 2012

\section{Werber · K. Stark}

Fachgebiet für gastroenterologische Infektionen, Zoonosen und tropische Infektionen,

Abteilung für Infektionsepidemiologie, Robert Koch-Institut, Berlin

\title{
Notwendigkeit einer systematischen Feintypisierung zur Ausbruchsfrüherkennung bei lebensmittelbedingten bakteriellen Erkrankungen
}

Der vorliegende Beitrag begründet aus dem Blickwinkel der Öffentlichen Gesundheit und Infektionsepidemiologie für Deutschland die Notwendigkeit einer flächendeckenden, systematischen Feintypisierungssurveillance für bestimmte bakterielle, häufig durch Lebensmittel übertragene Krankheitserreger. In den letzten Jahren wurde immer wieder deutlich, dass die bisher in Deutschland verfügbaren Instrumente zum frühzeitigen Erkennen von (insbesondere überregionalen) lebensmittelbedingten bakteriellen Krankheitsausbrüchen nicht ausreichen. Die mikrobiologischen Aspekte der Feintypisierung werden im sich anschließenden Beitrag von Flieger et al. dargestellt.

Akute infektiöse Gastroenteritiden bergen eine hohe Krankheitslast und werden - abhängig vom Erreger - zu einem beträchtlichen Teil durch kontaminierte Lebensmittel verursacht. Über Lebensmittel können viele Menschen auch über große geografische Entfernungen exponiert werden; daher treten Lebensmittel auch häufig als Erregervehikel bei Krankheitsausbrüchen in Erscheinung.

Labordiagnostische Nachweise von Krankheitserregern bilden die Grundlage für die Überwachung (Surveillance) meldepflichtiger Infektionserkrankungen gemäß dem Infektionsschutzgesetz (IfSG). Hierdurch können sowohl saisonale als auch langfristige Trends in der Inzidenz dieser Erkrankungen erkannt und be- obachtet werden. Das IfSG misst lebensmittelbedingten Infektionserkrankungen eine große Bedeutung zu. Dies wird verdeutlicht durch die Vielzahl der nach $\$ 7$ Absatz 1 IfSG meldepflichtigen Erregernachweise, die typische Krankheitserreger lebensmittelbedingter Erkrankungen betreffen, z. B. Salmonella enterica, Campylobacter spp., enterohämorrhagische E. coli (EHEC) etc. Darüber hinaus besteht eine Meldepflicht für den Verdacht auf bzw. die Erkrankung an einer mikrobiell bedingten Lebensmittelvergiftung oder einer infektiösen Gastroenteritis, wenn 2 oder mehr gleichartige Erkrankungen festgestellt werden, bei denen ein epidemischer Zusammenhang - mithin ein Krankheitsausbruch - vermutet wird.

Ein wesentliches Ziel der Surveillance lebensmittelbedingter Infektionserkrankungen ist das frühzeitige Erkennen von Krankheitsausbrüchen [1], sodass durch zielgerichtete Ermittlungen die Infektionsquelle - das kontaminierte Lebensmittel - identifiziert und abgestellt werden kann. Je früher ein Ausbruch erkannt wird, desto größer sind die Chancen, das verantwortliche Lebensmittel aufzudecken und Neuerkrankungen zu verhindern. Diverse lebensmittelbedingte Ausbrüche der letzten Jahre in Deutschland, unter anderem auch der massive EHEC O104-Ausbruch, haben vor Augen geführt, dass eine Verbesserung der Früherkennung von lebensmittelbedingten Ausbrüchen höchste Priorität haben muss.
Typische vom Öffentlichen Gesundheitsdienst (ÖGD) untersuchte lebensmittelbedingte Krankheitsausbrüche ereignen sich zumeist lokal nach gemeinschaftlicher Aufnahme von Lebensmitteln, z. B. im Rahmen von Festivitäten. Diese sowohl räumlich als auch zeitlich eng umschriebenen Krankheitsausbrüche sind für den ÖGD zumeist leicht zu erkennen (oft bereits durch die Betroffenen selbst), da es kurzfristig zu einer deutlichen Erhöhung der Krankheitszahl kommt.

Schwierig hingegen ist das Erkennen geografisch weiträumiger Krankheitsausbrüche, die durch ein überregional, manchmal sogar international, verteiltes kontaminiertes Lebensmittel hervorgerufen werden. Diese können, wie unter anderem der EHEC O104-Ausbruch gezeigt hat, zu sehr großen Erkrankungszahlen führen und zeitlich protrahiert auftreten [2, 3]. Da sie auf der lokalen Ebene (Land- bzw. Stadtkreis) selten zu einem sprunghaften Anstieg der Erkrankungszahlen führen, können sie von den Gesundheitsämtern häufig nicht bzw. erst spät bemerkt werden [4]. Überregionale Ausbrüche werden in Deutschland hauptsächlich auf 2 Wegen erkannt: zum einen über am RKI eingesetzte statistische Verfahren (Algorithmen), die erregerspezifisch eine überzufällige Häufung der Meldezahlen anzeigen (bundesweit oder in bestimmten Regionen oder in bestimmten demografischen Gruppen); zum anderen über Häufungen von be- 
stimmten ansonsten seltenen Erregertypen in Probenmaterialeinsendungen an das Nationale Referenzzentrum (NRZ) für Salmonellen und andere bakterielle Enteritiserreger. Mit der zunehmenden Zentralisierung der Produktion bzw. der Globalisierung im Handel von Lebensmitteln steigt das Risiko für geografisch disperse Ausbrüche, was die Notwendigkeit einer Surveillance verdeutlicht, die diese frühzeitig erkennt.

Ein besonders wertvolles und in anderen Ländern bewährtes Instrument zum frühzeitigen Erkennen von Krankheitsausbrüchen ist die systematische flächendeckende Feintypisierungssurveillance bestimmter bakterieller Krankheitserreger. Der Begriff „Feintypisierung“ meint hier die weitergehende Charakterisierung des Erregers möglichst auf der klonalen Ebene mittels moderner molekularbiologischer Methoden (s. Beitrag von Flieger et al. in dieser Ausgabe). Die Feintypisierungssurveillance stellt eine Erweiterung zur herkömmlichen Überwachung von Infektionserkrankungen dar. Dabei werden Krankheitserreger nach deren Primärnachweis zeitnah weitergehend charakterisiert, um die genetische Diversität auf der Subspeziesebene (möglichst auf der klonalen Ebene) zu erfassen und identische Erregerstämme zu entdecken. Diese Ergebnisse werden in die bestehende Surveillance der meldepflichtigen Infektionserkrankungen integriert. Zur Ausbruchsdetektion werden die Feintypisierungsergebnisse der Krankheitserreger kontinuierlich verglichen, um Gruppen (Cluster) identischer Erreger zu finden. Identische Ergebnisse in der Feintypisierung belegen eine enge phylogenetische Verwandtschaft der Erreger und geben Hinweise auf einen möglichen Infektionszusammenhang, mithin auf einen Krankheitsausbruch. Somit können Ausbrüche bereits deutlich früher erkannt und Untersuchungen zur Infektionsursache eingeleitet werden. Wenn für Erregerisolate von einigen wenigen Erkrankten identische Feintypisierungsergebnisse vorliegen, besteht ein Ausbruchsverdacht, dem gezielt nachgegangen werden kann. Unter Umständen begründet bereits der Nachweis identischer Erregerstämme bei 2 Erkrankten einen Ausbruchsverdacht. Die not- gedrungen derzeit in Deutschland vorherrschende Praxis, die Meldezahlen auf statistische Aberrationen zu prüfen, erfordert in der Regel eine deutlich höhere Zahl von Erkrankten, um einen Ausbruchsverdacht zu generieren.

Neben der zeitnahen Ausbruchsdetektion bietet eine Feintypisierungssurveillance weitere wichtige Vorteile:

1. Sensitive Ausbruchserkennung: Ausbrüche können erkannt werden, auch wenn sie nur zu einem geringen, statistisch (noch) nicht signifikanten Anstieg der Erkrankungsbzw. Meldezahlen führen. Dies ist wichtig bei Erkrankungen mit hoher Hintergrundrate bzw. bei geografisch sehr diffuser Fallverteilung, also bei überregionalen Ausbrüchen.

2. Spezifischere Ausbruchsuntersuchung: Zum Ausbruch gehörende Erkrankungen können durch die Feintypisierung definiert werden. Der Ausbruch umfasst somit nicht zwingend alle Erkrankungen, die in einem zeitlichen und räumlichen Zusammenhang stehen. Dies führt zu einer genaueren Expositionscharakterisierung im Rahmen der epidemiologischen Untersuchungen, da keine Expositionserhebung bei „Hintergrundfällen“ erfolgt, die zwar im zeitlichen und/oder räumlichen, aber nicht im epidemischen Zusammenhang stehen. Als Folge führt dies zu einer erhöhten Aufklärungsrate und Aufklärungsgeschwindigkeit von Ausbrüchen.

3. Spezifischerer Einsatz personeller Ressourcen:

a) Nicht zum Ausbruch gehörende Fälle (d. h. in der Feintypisierung frühzeitiger Nachweis eines anderen Erregerstammes als der des Ausbruchsstammes) werden nicht in die Ausbruchsuntersuchung einbezogen (s. oben).

b) Eine Ausbruchsuntersuchung kann unterbleiben, wenn statistische Algorithmen zwar eine überzufällige Erhöhung der Erkrankungszahlen anzeigen, jedoch aufgrund unterschiedlicher Feintypisierungsergebnisse bei den Erkrankten auf eine nur zufälli- ge Erhöhung („Pseudoausbruch“) geschlossen werden kann.

In anderen Ländern, vor allem in den USA, hat sich eine Feintypisierungssurveillance außerordentlich bewährt [5]. Grundlage des in den USA etablierten „PulseNet“ [6] ist die zeitnahe Typisierung ausgewählter lebensmittelassoziierter Erreger durch Labore des Öffentlichen Gesundheitsdienstes. Diese über die USA verteilten Labore untersuchen eingesandte Isolate unverzüglich nach standardisierten Kriterien mittels Pulsfeldgelelektrophorese (PFGE) und senden die durch diese Untersuchungsmethode entstehenden Muster („genetischer Fingerabdruck") an eine dynamische Datenbank. Sieben der 10 größten Ausbrüche des letzten Jahrzehnts in den USA wären ohne PulseNet nicht erkannt bzw. untersucht worden (John Besser, CDC, persönliche Mitteilung). Dabei wurden auch immer wieder neue Lebensmittelvehikel für beispielsweise Salmonellen entdeckt, wie z. B. Erdnussbutter [7] oder gehacktes Thunfischfleisch in Sushi. Dieses Labornetzwerk schließt auch staatliche Labore der Lebensmittelüberwachung sowie mittlerweile Labore aus anderen Ländern und sogar Kontinenten ein [8].

Dass ein solches Feintypisierungssystem auch in Deutschland geeignet ist, Krankheitsausbrüche sensitiv zu erkennen, konnte in einem vom NRZ koordinierten Projekt gezeigt werden, in dem ca. 40 niedergelassene Labore dem NRZ EHEC-positive Proben weiterleiteten, um sie dort weiter zu charakterisieren. Dadurch konnte ein Ausbruch durch EHEC O26:H11 entdeckt werden, der zu 11 Infektionen in 3 Bundesländern führte. Als wahrscheinliche Ursache für den Ausbruch wurde ein Rindfleischprodukt identifiziert [9].

Eine Feintypisierungssurveillance erscheint auch kosteneffizient. Nach Schätzungen der US-amerikanischen Centers for Disease Control and Prevention müssten zur Amortisation beispielsweise einer EHEC O157:H7-Feintypisierungssurveillance in den ersten 5 Jahren lediglich 21 Erkrankungen verhindert werden [10]. Hier wurden die Kosten der laborgestützten Überwachung einschließ- 
lich der Kosten für die Ausbruchsuntersuchung den durchschnittlichen Kosten einer EHEC O157:H7-Erkrankung gegenübergestellt.

Damit eine Feintypisierungssurveillance zeitnah und sensitiv Krankheitsausbrüche erkennt, muss ein möglichst hoher Anteil hierfür geeigneter Laborproben schnell in das System der Feintypisierungssurveillance eingespeist werden (Weiterleitung der Erregerisolate bzw. Proben zur Erregeranzucht aus den Primärlaboren an die Labore, die die Feintypisierung nach standardisierten Methoden durchführen, z. B. Nationales Referenzzentrum, Konsiliarlabore, ggf. Landeslabore). Die bisherigen Erfahrungen zeigen, dass die Weiterleitung solcher Proben auf freiwilliger Basis derzeit nicht zu einem ausreichend hohen Probenaufkommen führt. Hier sind Weiterentwicklungen erforderlich, um ein möglichst vollzähliges Probenaufkommen für die Feintypisierung $\mathrm{zu}$ realisieren.

Darüber hinaus ist ein System erforderlich, das die rasche Feintypisierung und - auf der überregionalen Ebene die Auswertung der Daten (Datenbankanalysen, Verknüpfung mit Surveillancedaten) sicherstellt. Entscheidend hierfür ist die Wahl einer geeigneten Feintypisierungsmethode, wobei die Methode mit der höchsten Diskriminierung zwischen Erregerisolaten nicht notwendigerweise die bevorzugte Methode ist. Von zentraler Bedeutung ist vielmehr die Übereinstimmung zwischen den mikrobiologischen Ergebnissen und der epidemiologischen Situation, d. h., identische Ergebnisse bei der Feintypisierung deuten auf eine gemeinsame Infektionsursache hin, divergente Ergebnisse machen hingegen eine gemeinsame Infektionsursache unwahrscheinlich. Ein weiteres wichtiges Kriterium für die Auswahl der geeigneten molekularbiologischen Methode zur Feintypisierung ist eine gute Standardisierbarkeit und Vergleichbarkeit der Ergebnisse zwischen den Laboren auf nationaler und internationaler Ebene. Die Typisierungsinformationen müssen den betroffenen Behörden auf Landesebene und Lokalebene zeitnah zur Verfügung gestellt werden, damit sie bei re-

Bundesgesundheitsbl 2013 · 56:38-41 DOI 10.1007/s00103-012-1591-3

(c) Springer-Verlag Berlin Heidelberg 2012

\section{Werber $\cdot$ K. Stark}

\section{Notwendigkeit einer systematischen}

Feintypisierung zur Ausbruchsfrüherkennung bei lebensmittelbedingten bakteriellen Erkrankungen

\section{Zusammenfassung}

Ein wesentliches Ziel der Surveillance lebensmittelbedingter Infektionserkrankungen ist das frühzeitige Erkennen von Krankheitsausbrüchen. In diesem Zusammenhang ist eine systematische flächendeckende Feintypisierungssurveillance ein besonders wertvolles Instrument für ausgewählte bakterielle Erreger, wie z. B. enterohämorrhagische E. coli (EHEC), Listeria monocytogenes, oder bestimmte Salmonellen-Serovare. Mit molekularen Methoden der Feintypisierung werden Erregerisolate von Patienten weitergehend und möglichst auf der klonalen Ebene charakterisiert, um identische Erregerstäm- me und damit Ausbrüche zu entdecken und Erkrankte einem bestimmten Ausbruchsgeschehen zuzuordnen. In dem hier vorgelegten Plädoyer wird aus Sicht der Infektionsepidemiologie begründet, warum in Deutschland für ausgewählte bakterielle Erreger eine systematische flächendeckende Feintypisierungssurveillance notwendig ist und welche entscheidenden Vorteile sie bietet.

Schlüsselwörter Lebensmittelbedingte Infektionen . Ausbrüche · Feintypisierung · Surveillance . Escherichia coli

\section{The need for systematic molecular subtyping surveillance to timely detect food-borne outbreaks}

\section{Abstract}

Timely outbreak detection is a major objective of the surveillance of food-borne infections. In this regard molecular subtyping is a very useful tool for several bacterial pathogens, e.g. enterohemorrhagic $E$. coli (EHEC), Listeria monocytogenes and various serotypes of non-typhoidal Salmonella. The basis is the characterization of patient isolates by molecular methods, preferably at the clonal level. The goal is to find groups of identical isolates which may indicate that they share a com- mon origin, i.e. they might belong to an outbreak. In this article we put forward strong reasons why a systematic nationwide molecular subtyping surveillance is needed for selected bacterial pathogens in Germany.

Keywords Food-borne diseases - Disease outbreaks . Molecular typing · Public health · Shiga-like toxin - Escherichia coli gional begrenzten Ausbrüchen selbst aktiv werden können. Insgesamt stellt die notwendige Etablierung einer flächendeckenden, systematischen Feintypisierungssurveillance für Deutschland eine große Herausforderung dar, für die zusätzliche Ressourcen bereitstehen müssen. Wichtig ist eine gute Vernetzung und klare Rollenverteilung zwischen der auf therapeutische Belange ausgerichteten Primärdiagnostik und der für die Öffentliche Gesundheit bedeutsamen Detailcharakterisierung (z. B. Feintypisierung) von Krankheitserregern. Für die optimale Umsetzung einer Feintypisierungssurveillance sollten standardisierte Verfahrensweisen konzeptualisiert werden. Dies betrifft vor allem Details zur zeitnahen Gewinnung und Wei- terleitung möglichst vieler Proben (bzw. Isolate) und die Datenauswertung (zum Stand der Fortentwicklung entsprechender Konzepte s. den Beitrag von Flieger et al. in dieser Ausgabe).

Wird ein Ausbruch erkannt, muss als nächster Schritt sichergestellt sein, dass er zeitnah in koordinierter Weise untersucht wird. Handelt es sich um ein überregionales Geschehen, also eines, bei der Menschen in unterschiedlichen Landbzw. Stadtkreisen exponiert waren, sollte die Ausbruchsuntersuchung von den obersten Landesgesundheitsbehörden (bei Ausbrüchen innerhalb eines Bundeslandes) bzw. vom Robert Koch-Institut (zumindest bei Bundesland-übergreifenden Krankheitsausbrüchen) koordiniert werden. 


\section{Korrespondenzadresse}

\section{PD Dr. D. Werber}

Fachgebiet für gastroenterologische Infektionen,

Zoonosen und tropische Infektionen,

Abteilung für Infektionsepidemiologie,

Robert Koch-Institut

DGZ-Ring 1, 13086 Berlin

werberd@rki.de

Interessenkonflikt. Der korrespondierende Autor gibt für sich und seinen Koautor an, dass kein Interessenkonflikt besteht.

\section{Literatur}

1. Werber D, Kramer MH, Buchholz U et al (2006) Surveillance of infectious foodborne diseases after enactment of the German Protection against Infection Act in 2001. Potentials and requirements. Bundesgesundheitsbl Gesundheitsforsch Gesundheitsschutz 49:1020-1026

2. Frank C, Werber D, Cramer JP et al (2011) Epidemic profile of shiga-toxin-producing Escherichia coli 0104:H4 Outbreak in Germany. N Engl J Med 365(19):1771-1780

3. Buchholz U, Bernard H, Werber D et al (2011) German outbreak of Escherichia coli 0104:H4 Associated with sprouts. N Engl J Med 365(19):1763-1770

4. Werber D, Dreesman J, Feil F et al (2005) International outbreak of Salmonella Oranienburg due to German chocolate. BMC Infect Dis 5:7

5. Tauxe RV (2006) Molecular subtyping and the transformation of public health. Foodborne Pathog Dis 3:4-8

6. Swaminathan B, Barrett TJ, Hunter SB, Tauxe RV (2001) PulseNet: the molecular subtyping network for foodborne bacterial disease surveillance, United States. Emerg Infect Dis 7:382-389

7. Sheth AN, Hoekstra M, Patel N et al (2011) A national outbreak of Salmonella serotype Tennessee infections from contaminated peanut butter: a new food vehicle for salmonellosis in the United States. Clin Infect Dis 53:356-362

8. Swaminathan B, Gerner-Smidt P, Ng LK et al (2006) Building PulseNet International: an interconnected system of laboratory networks to facilitate timely public health recognition and response to foodborne disease outbreaks and emerging foodborne diseases. Foodborne Pathog Dis 3:36-50

9. Werber D, Fruth A, Liesegang A et al (2002) A multistate outbreak of Shiga toxin-producing Escherichia coli 026:H11 infections in Germany, detected by molecular subtyping surveillance. J Infect Dis 186:419-422

10. Elbasha EH, Fitzsimmons TD, Meltzer MI (2000) Costs and benefits of a subtype-specific surveillance system for identifying Escherichia coli 0157:H7 outbreaks. Emerg Infect Dis 6:293-297

\section{Aktuelle Schätzung der Zahl von HIV-Infektionen in Deutschland}

Aktuell leben nach einer aktuellen Schätzung des Robert Koch-Instituts (RKI) in

Deutschland etwa 78.000 Menschen mit einer HIV-Infektion. Die Gesamtzahl der mit HIV lebenden Menschen steigt seit Mitte der 90er Jahre, und wird auch in den kommenden Jahren weiter ansteigen, da seit der Verfügbarkeit wirksamer Therapien für die HIV-Infektion die Zahl der jährlichen Neuinfektionen höher ist als die Zahl der Todesfälle. Das RKI schätzt, dass mittlerweile etwa 50.000 Menschen in Deutschland eine HIV-Therapie erhalten und von den weiteren ca. 28.000 Infizierten etwa 14.000 Betroffene noch nichts von ihrer Infektion wissen.

Die Zahl der HIV-Neuinfektionen in Deutschland hat nach einem Anstieg zu Beginn des vergangenen Jahrzehnts ab 2004/2005 ein Plateau erreicht, auf dem die Zahl der Neuinfektionen seitdem mit relativ geringen Auf- und Abwärtsbewegungen schwankt. Für das Jahr 2012 wurden rund $3.400 \mathrm{Neu}-$ infektionen geschätzt (2011: 3.300), die Zahl der Todesfälle betrug unverändert gegenüber dem Vorjahr etwa 550. Diese Zahlen unterstreichen, dass die Anstrengungen zur Vermeidung von Infektionen weiterhin hohe Priorität und ausreichende Finanzierung erfordern.

Die am stärksten betroffene Gruppe sind weiterhin Männer, die Sex mit Männern haben (MSM). Von den 78.000 HIV-Infizierten in Deutschland zählen etwa 51.000 zu dieser Gruppe. Bei MSM kam es Ende der 90er Jahre zu einer deutlichen Zunahme von Neuinfektionen, die etwa ab 2004 in eine Plateau-Phase übergegangen ist. Dies steht vermutlich auch in Zusammenhang mit der seit Ende der 90er Jahre deutlich gestiegenen Verbreitung anderer sexuell übertragbarer Infektionen, die eine Übertragung von HIV begünstigen. Eine schnelle Diagnose und Behandlung von anderen sexuell übertragbaren Infektionen (bspw. Syphilis) kann daher dem Anstieg der HIV-Neuinfektionen entgegenwirken.
Die Zahl der HIV-Neuinfektionen ist zu unterscheiden von der Zahl der (dem RKI gemeldeten) HIV-Neudiagnosen. Die HIV-Infektion, die oftmals unbemerkt erfolgt, und der Test, mit dem eine Infektion nachgewiesen wird, können zeitlich weit auseinander liegen. Die Zahl der Neuinfektionen kann daher nur geschätzt werden. Die aktuelle Schätzung ist nicht direkt mit den Schätzungen vorangegangener Jahre vergleichbar, da jede neue Modellierung aufgrund zum Teil veränderter Annahmen und Methoden für den gesamten Zeitverlauf neue Werte ergibt.

Quelle: Robert Koch-Institut, Berlin, www.rki.de 